\title{
Erratum to: Transcriptome profile reveals AMPA receptor dysfunction in the hippocampus of the Rsk2-knockout mice, an animal model of Coffin-Lowry syndrome
}

Tahir Mehmood $\cdot$ Anne Schneider $\cdot$ Jérémie Sibille $\cdot$ Patricia Marques Pereira

Solange Pannetier • Mohamed Raafet Ammar • Doulaye Dembele ·

Christelle Thibault-Carpentier • Nathalie Rouach • André Hanauer

Published online: 17 December 2010

(C) Springer-Verlag 2010

\section{Erratum to: Hum Genet}

DOI 10.1007/s00439-010-0918-0

Unfortunately, one of the co-author names has been misspelled in the online published article. The third author name should be changed from Jérémie Sibillec to Jérémie Sibille.

The online version of the original article can be found under doi:10.1007/s00439-010-0918-0.

T. Mehmood · A. Schneider · P. Marques Pereira .

S. Pannetier - M. R. Ammar - A. Hanauer $(\bowtie)$

Department of Translational Medicine and Neurogenetics, Institut de Génétique et de Biologie Moléculaire et Cellulaire (IGBMC), Institut National de Santé et de Recherche Médicale (INSERM) U964/Centre National de Recherche Scientifique (CNRS) UMR 7104/Université de Strasbourg,

67404 Illkirch, France

e-mail: Andre.HANAUER@igbmc.fr

\section{T. Mehmood}

Department of Chemistry, University of Sargodha,

Sargodha 40100, Pakistan

D. Dembele $\cdot$ C. Thibault-Carpentier

Microarray and Sequencing Platform, Institut de Génétique et de Biologie Moléculaire et Cellulaire (IGBMC), Institut National de Santé et de Recherche Médicale (INSERM) U964/Centre

National de Recherche Scientifique (CNRS)

UMR 7104/Université de Strasbourg, 67404 Illkirch, France

J. Sibille $\cdot$ N. Rouach

Collège de France, 75005 Paris, France 